\title{
Priming effects on temporal order judgments about words: Perceived temporal priority or response bias?
}

\author{
BRYAN R. BURNHAM, JAMES H. NEELY, and PATRICK A. O'CONNOR \\ University at Albany, State University of New York, New York
}

\begin{abstract}
In a temporal order judgment task, in which observers select which of two words appeared first, Stolz (1999) found that observers were more likely to select the word that had been semantically primed. Using repetition priming, we replicated Stolz's finding and extended her results by demonstrating that the effect was due to both (1) repetition priming causing the primed item to be perceived as having occurred earlier and (2) a response bias to guess the repetition primed item as the correct response. We discuss our new finding that priming induces an attentional precedence effect in the context of previous research suggesting that exogenous spatial cuing induces an attentional precedence effect but identity or semantic priming may not.
\end{abstract}

One of Titchener's (1908) seven fundamental laws of attention was the law of prior entry: "The object of attention comes to consciousness more quickly than the objects which we are not attending to" (p. 251). Support for this comes from the temporal order judgment (TOJ) task, in which observers choose which of two stimuli has occurred first. In that task, exogenously cuing attention to a location in visual space or directing attention toward a particular sensory modality causes observers to choose the stimulus that has appeared at that location or in that sensory modality as having occurred earlier than a stimulus that has appeared at an uncued location (e.g., Scharlau, 2002, 2004 ) or in an "uncued" sensory modality (Spence, Shore, \& Klein, 2001).

Here, we focus on TOJ tasks with visual stimuli. In one version of this task (see Scharlau, 2004), observers see two shapes (e.g., a diamond and a square), one to the left and one to the right of central fixation, and judge which one has appeared first. Prior to their onsets, a single masked "prime" appears briefly in the same location as one of the stimuli, and it is typically identical to this "primed" item. When the stimulus onset asynchrony (SOA) between the two shapes is short (including $0 \mathrm{msec}$ ) an observer often reports that the primed shape appeared first. However, as has been pointed out with respect to a variety of TOJ paradigms (see Scharlau, 2002; Scharlau \& Neumann, 2003; Spence et al., 2001; Stelmach \& Herdman, 1991), it is unclear whether such effects are caused by attention's being

The data from a subset of the participants tested herein were presented at the 16th annual convention of the American Psychological Society, May 2004, Chicago. We thank Tram Neill for helpful commentary, Peter B. Walker and Jeffrey D. Johnson for programming assistance, and Jiah Pearson-Leary and Joy Perkett for helping with data collection. Address correspondence to J. H. Neely, Department of Psychology, Social Sciences 369, University at Albany, Albany, New York 12222 (e-mail: jn562@albany.edu). drawn to (1) the primed item's identity or modality or (2) its location via exogenous cuing (e.g., Posner, 1980). Because Scharlau's (2004) study showed priming to be of the same magnitude whether a shape was exogenously cued by a prime having the same or a different identity (see also Scharlau, 2002; Scharlau \& Neumann, 2003), Scharlau's effect was probably due solely to exogenous spatial cuing and not priming.

A critical issue is whether this "priming"/exogenous cuing effect on TOJs is due to (1) enhanced visual processing of the primed shape causing it to be perceived as having occurred earlier or (2) a response bias to guess as the "correct" response the more highly activated/attended primed shape when the observer has no distinct perception of temporal order (cf. Scharlau, 2004; Spence et al., 2001). Although this issue has been examined rather extensively (Scharlau, 2004; Spence et al., 2001), we focus on a recent study by Scharlau (2004, Experiment 3). In one block, Scharlau's observers made TOJs by responding which of two shapes appeared first (TOJ-1 st instructions) and in another block, which appeared second (TOJ-2nd instructions). The logic behind this instructional manipulation is as follows (cf. Jacoby's [1991] method-ofopposition procedure): For TOJ-1st instructions, priming's effect on response bias and perceived temporal priority operate in concert, both increasing the probability of responding with the primed shape. However, for TOJ-2nd instructions, priming and bias operate in opposition. That is, enhanced perceptual processing of the primed shape when it actually has appeared first would also increase the probability of correctly responding that the unprimed shape appeared second, whereas the response bias would cause the primed shape to be incorrectly guessed as having occurred second.

Consider three possible outcomes for trials with asynchronous TOJ items: ${ }^{1}$ (1) If the "priming" effect on TOJs were due entirely to enhanced perceptual processing, then 
priming should be identical for both instructional sets (i.e., a significant main effect of priming and a null priming $\times$ instructions interaction). (2) If response bias were the sole cause of "priming" on TOJs, priming for TOJ-2nd instructions should be of the same absolute magnitude as priming for TOJ-1st instructions, but negative (i.e., a null main effect of priming and a significant priming $\times$ instructions interaction). (3) If response bias and enhanced perceptual processing both contribute, for TOJ-2nd instructions the response bias effect would reduce or eliminate "priming" in comparison with TOJ-1st instructions (i.e., the main effect of priming and the priming $\times$ instructions interaction would both be significant). Because "priming" effects were nearly identical for both instructional sets (Outcome 1), Scharlau's (2004) data suggest that her "priming" effects for TOJs were due to enhanced perceptual processing of the primed shape, with response bias having no measurable impact. However, because "identity priming" had no measurable effect, the data only show that exogenous cuing causes an attentional precedence effect; they are moot regarding whether identity priming, when it occurs, affects TOJs via enhanced perceptual processing, a response bias, or both.

The foregoing analysis raises the following important question: In the absence of exogenous cuing, will a centrally presented prime affect TOJs, and if so, would the effect be due to enhanced perceptual processing, a response bias, or both? Relevant data are reported by Stolz (1999) from experiments wherein two unrelated TOJ words, one to each side of fixation, were preceded by a spatially centered, briefly presented masked prime that was semantically related to one of the TOJ words. When the SOA between the TOJ words was 15 or $0 \mathrm{msec}$, the semantically primed word was selected more often as having occurred first. This shows that TOJs can be affected by priming the meaning of a TOJ item. Stolz explained this effect as being due to the primed word's being given attentional precedence over the unprimed word, causing it to be perceived as having occurred first. However, it could also be that at very short SOAs between the TOJ words, observers were biased to guess as the "correct" response the more highly activated, semantically primed word, even though it was not actually perceived as having occurred earlier (cf. Scharlau, 2004).

Indeed, it is plausible that Stolz's (1999) results may have been completely due to a response bias. The rationale is as follows: Exogenous cuing is known to affect both the rate of perceptual uptake (because its effects interact with those of stimulus intensity as in Stolz \& Stevanovski, 2004) and an item's perceived perceptual priority (Scharlau \& Neumann, 2003; Spence et al., 2001; Stelmach \& Herdman, 1991). This, along with Scharlau's (2004) method-of-opposition data, suggests that exogenous cuing influences TOJs by enhancing perceptual processing of the primed item. Given this, by Sternberg's (1969) additivefactors logic, if Stolz's priming effect on TOJs was also due to enhanced perceptual processing, one would expect priming to interact with exogenous cuing. Unfortunately,
Scharlau's (2004) TOJ results do not permit a test of this prediction because identity priming had no discernible effect on performance (perhaps because the TOJ shapes received massive across-trial repetition priming). However, Stolz and Stevanovski (2004, Experiment 2), using a lexical decision task, demonstrated that (1) semantic priming and exogenous cuing do not interact (despite sizeable main effects of both variables on performance) and (2) stimulus intensity effects interact with exogenous cuing effects but not with semantic priming effects. ${ }^{2}$ This pattern of data suggests that priming and exogenous cuing affect different mechanisms, which in turn implies that Stolz's priming effect on TOJs was not due to priming's having affected the perception of temporal priority by enhancing perceptual processing, as seems true for exogenous cuing. Rather, priming likely influenced a later processing stage such as decisional response bias.

In the present experiment, we examined the degree to which priming affects TOJs via enhancing perceptual processing or through producing a response bias. Our experiment, with three different TOJ SOAs (0, 17, and $200 \mathrm{msec})$, differed from Stolz's (1999) experiments in two important ways: (1) Because repetition priming typically produces larger effects than does semantic priming (see, e.g., Dannenbring \& Briand, 1982), we used the former, hoping to maximize our chances of detecting both perceptual enhancement and response bias effects. (2) More important, we used Scharlau's (2004, Experiment 3) instructional manipulation to determine, per the logic of the method of opposition, whether the priming effect on TOJs is due to response bias, enhanced perceptual processing, or both.

\section{METHOD}

\section{Participants}

Eighty-two native English speaking undergraduates from the University at Albany reporting normal or corrected-to-normal vision participated for course credit. Data from 2 participants were discarded due to a failure to complete the task. The analyses are based on the remaining 80 participants (40 in each instructional group).

\section{Design}

The main part of the design was a 2 (TOJ Instructions: choose 1 st presented word vs. choose 2 nd presented word) $\times 2$ (TOJ SOA: $200 \mathrm{msec}, 17 \mathrm{msec}) \times 2$ (primed word location: top vs. bottom) $\times$ 2 (1st presented word location: top vs. bottom) mixed-factor factorial, with TOJ instruction a between-participants factor and all other factors within participants. The additional condition, a $0-\mathrm{msec}$ TOJ SOA, was analyzed separately because the primed and the unprimed word appeared simultaneously.

\section{Stimuli}

Two hundred forty pairs of unrelated words were constructed. One word from each pair served as a prime, and both words served as the two TOJ words on a given trial. Forty of these pairs were used for practice and warm-up trials, and the remaining 200 pairs were used for critical trials from which the data are reported. These 200 critical pairs were subdivided into five unique sets of 40 pairs that received a fixed assignment to one of five conditions: the 0 -msec SOA condition or one of the other four conditions created by the 2 (SOA: 200 vs. $17 \mathrm{msec}) \times 2$ (1st presented target's location: top vs. bottom) design. Across four counterbalancing lists (each given 
to 10 participants in each instructional group), each word in each pair occurred once each in the primed and unprimed conditions when it appeared above fixation and below fixation. Thus, averaged across the four lists, differences in performance across SOAs could potentially be due to item differences, but the effects of the other variables (crucially priming and instructions) could not. The forward and backward masks consisted of a row of 10 \#s and $10 \mathrm{Xs}$, respectively. All stimuli were presented in white lowercase letters (MEL color 15) on a black background. Although masking did not preclude awareness of the prime's identity on all trials, this does not affect our interpretation of the results.

\section{Procedure}

The experiment was run with Micro Experimental Laboratory, Version 2 (Schneider, 1995) on a Gateway 2000 computer with a 15 -in. monitor. Forty participants were instructed to select the word (i.e., top or bottom) that appeared first, and the other 40, the word that appeared second. All were told to favor accuracy over speed and that the stimuli presented before the two TOJ words were irrelevant to the task. Sixteen practice trials (without a prime) and 24 warm-up trials (with a prime) were followed by the 200 experimental trials.

On each trial, a 500-msec fixation cross $(+)$ appeared in the center of the screen and was replaced by the 500 -msec forward mask. For the 24 warm-up and 200 critical trials, a $100-\mathrm{msec}$ word prime was then immediately presented, whereas a $100-\mathrm{msec}$ row of 10 $\mathrm{Xs}$ was presented for the initial 16 practice trials. The prime (or row of Xs) was immediately replaced by the $100-\mathrm{msec}$ backward mask. Upon the backward mask's offset, one of the two TOJ words appeared $1.72^{\circ}$ above or below fixation (the viewing distance was approximately $50 \mathrm{~cm}$ ). The second word appeared at the other location $0 \mathrm{msec}$ (i.e., simultaneously with the first word), $17 \mathrm{msec}$, or $200 \mathrm{msec}$ after the first word's onset. Both words disappeared upon response. For TOJ responses, the upper and lower words were assigned to the "C" and "M" keys of the keyboard, respectively. The intertrial interval was $1,000 \mathrm{msec}$.

\section{RESULTS}

The data in Table 1 show the proportions of accurate TOJs for the 17- and 200-msec SOAs. Thus, for the TOJ1st and TOJ-2nd instructions, the entries are the probabilities that the first-presented or second-presented word, respectively, was correctly selected, when that word was primed or unprimed. (The format for the 0-msec SOA entries is discussed later.) All effects reported as significant were associated with $p<.05$, two-tailed.

\section{Main Analyses Excluding the 0-msec SOA}

The comparisons to follow are based on the results of a 2 (SOA: 200, $17 \mathrm{msec}) \times 2$ (priming: primed, unprimed) $\times$ 2 (TOJ instructions: choose first or second word) mixedfactor ANOVA with TOJ instructions the only betweenparticipants factor. At the 200-msec SOA, TOJs were reasonably accurate $(.77 \pm .05$; the number after the " \pm " is the $95 \%$ confidence interval). Correct perceptions were $.24 \pm .05$ greater (Cohen's $d_{\mathrm{IGD}}=1.14, d_{\mathrm{CD}}=1.58$; see the Table 1 note for explanation of the two $d$ s) for the $200-\mathrm{msec}$ SOA than for the 17-msec SOA (.54 . .01), as indicated by a significant main effect of SOA $\left[F(1,78)=98.93, M S_{\mathrm{e}}=\right.$ .045]. More important, averaged across instructions and $\mathrm{SOA}$, the $.23 \pm .10$ priming effect $\left(d_{\mathrm{IGD}}=.81, d_{\mathrm{CD}}=.65\right)$ was significant $\left[F(1,78)=19.98, M S_{\mathrm{e}}=.207\right]$, due to more accurate responding for primed words $(.77 \pm .05)$ than for unprimed words $(.54 \pm .07)$. Furthermore, the significant priming effect at the 17-msec SOA (.26士.12) $\left[t(79)=4.43, S E=.058 ; d_{\mathrm{IGD}}=.96, d_{\mathrm{CD}}=.70\right] \mathrm{was}$ larger than the significant priming effect at the 200-msec $\mathrm{SOA}(.20 \pm .11)\left[t(79)=3.66, S E=.054 ; d_{\mathrm{IGD}}=.59\right.$, $\left.d_{\mathrm{CD}}=.58\right]$, as shown by the significant $\mathrm{SOA} \times$ priming interaction $\left[F(1,78)=7.59, M S_{\mathrm{e}}=.009\right]$. This finding replicates Stolz's (1999) results showing greater priming for more difficult temporal discriminations, but this by itself does not distinguish between perceptual precedence and response bias as mediators of the priming effect.

Of most importance was whether priming differed significantly between the Instructional groups. A significant instructions $\times$ priming interaction confirmed that the robust $.42 \pm .13$ priming effect $[t(39)=6.60, S E=.063$; $\left.d_{\mathrm{IGD}}=1.98, d_{\mathrm{CD}}=1.48\right]$ for the TOJ-1st group was statistically greater than the nonsignificant $.04 \pm .16$ priming effect $\left[t(39)<1 ; d_{\mathrm{IGD}}=.12, d_{\mathrm{CD}}=.10\right]$ for the TOJ-2nd group $\left[F(1,78)=14.17, M S_{\mathrm{e}}=.207 ; d_{\mathrm{CD}}=2.24\right]$. The three-way SOA $\times$ instructions $\times$ priming interaction did not reach conventional significance $[F(1,78)=2.85$, $\left.M S_{\mathrm{e}}=.009, p=.095\right]$. As outlined in the introduction, the main effect of priming combined with the instructions $\times$ priming interaction strongly suggests that priming was produced by both an attentional precedence effect and a response bias to choose the primed item.

\section{0-msec SOA Data}

The entries in Table 1 for the 0 -msec SOA show, for each instructional group, the probabilities that participants chose the primed item as the "correct" response. ${ }^{3}$ The $.75 \pm .07$ probability of choosing the primed item in the TOJ-1st group was significantly greater than chance (.50). This could be due to a perceptual precedence effect, a bias to choose the primed item, or both. However, had a bias alone produced the results, the TOJ-2nd group should have chosen the primed item equally as often as the

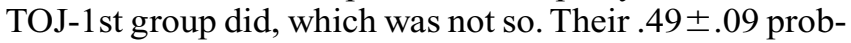
ability of choosing the primed item did not differ from chance but was significantly lower than that for the TOJ1 st group $\left[t(78)=4.74, S E=.055 ; d_{\mathrm{CD}}=.71\right]$. However, had these results been due solely to an attentional precedence effect, the TOJ-2nd group's mean should have been .25 - namely, as far below chance as the TOJ-1st group's mean was above chance-which clearly did not obtain. Hence, these data also show that priming affects both attentional precedence and response bias.

\section{DISCUSSION}

Our results replicate, and extend to repetition priming, Stolz's (1999) observation that a semantically primed word in a pair of TOJ words is more likely to be selected as having appeared first. More important, to our knowledge this is the first demonstration that priming the identity/ meaning of an item affects TOJs by actually facilitating the perception of the primed item's temporal priority, as Stolz (1999) argued. However, our data further show that 
Table 1

Proportion of Times the First-Presented Word Was Correctly Perceived as Having Occurred First for the 200-msec and 17-msec SOAs, As a Function of Response Instruction and Whether the First-Presented Word Was Primed or Unprimed, and the Proportion of Times the Primed Item Was Selected at the 0-msec SOA

\begin{tabular}{|c|c|c|c|c|c|c|c|c|c|}
\hline \multirow[b]{3}{*}{ SOA } & \multirow[b]{3}{*}{$(\mathrm{msec})$} & \multicolumn{8}{|c|}{ Response Instruction } \\
\hline & & \multicolumn{4}{|c|}{ TOJ 1st } & \multicolumn{4}{|c|}{ TOJ 2nd } \\
\hline & & $\begin{array}{l}\text { Primed } \\
\text { Word 1st }\end{array}$ & & $\begin{array}{l}\text { Unprimed } \\
\text { Word 1st }\end{array}$ & $\begin{array}{c}\text { Priming } \\
\text { Effect }\end{array}$ & $\begin{array}{c}\text { Primed } \\
\text { Word 1st }\end{array}$ & & $\begin{array}{c}\text { Unprimed } \\
\text { Word 1st }\end{array}$ & $\begin{array}{c}\text { Priming } \\
\text { Effect }\end{array}$ \\
\hline 200 & $\begin{array}{l}M \\
d_{\mathrm{IGD}} / d_{\mathrm{CD}^{\mathrm{a}}} \\
\# \mathrm{Os}^{\mathrm{b}}\end{array}$ & $\begin{array}{c}.95^{\dagger} \\
9.20 \\
40 / 40^{*}\end{array}$ & & $\begin{array}{c}.58 \\
.28 \\
24 / 40\end{array}$ & $\begin{array}{c}.37 \pm .14^{*} \\
1.31 / 1.23 \\
30 / 38^{*}\end{array}$ & $\begin{array}{c}.79^{\dagger} \\
1.13 \\
32 / 40^{*}\end{array}$ & & $\begin{array}{c}.76^{\dagger} \\
1.07 \\
33 / 40^{*}\end{array}$ & $\begin{array}{c}.03 \pm .15 \\
.07 / .08 \\
17 / 31\end{array}$ \\
\hline 17 & $\begin{array}{l}M \\
d_{\mathrm{IGD}} / d_{\mathrm{CD}}{ }^{\mathrm{b}} \\
\# \mathrm{Os}\end{array}$ & $\begin{array}{c}.75^{\dagger} \\
1.93 \\
37 / 40^{*}\end{array}$ & & $\begin{array}{c}.29^{\dagger} \\
1.34 \\
30 / 40^{*}\end{array}$ & $\begin{array}{c}.47 \pm .13^{*} \\
2.28 / 1.66 \\
34 / 39^{*}\end{array}$ & $\begin{array}{c}.57 \\
.36 \\
29 / 40^{*}\end{array}$ & & $\begin{array}{c}.53 \\
.13 \\
22 / 38\end{array}$ & $\begin{array}{c}.04 \pm .17 \\
.17 / .12 \\
24 / 39\end{array}$ \\
\hline 0 & $\begin{array}{l}M \\
d_{\mathrm{IGD}} / d_{\mathrm{CD}} \mathrm{b} \\
\# \mathrm{Os}\end{array}$ & $\begin{array}{l}- \\
- \\
-\end{array}$ & $\begin{array}{c}.75^{\dagger} \\
1.69 \\
33 / 37^{*}\end{array}$ & $\begin{array}{l}- \\
- \\
-\end{array}$ & $\begin{array}{l}- \\
- \\
-\end{array}$ & $\begin{array}{l}- \\
- \\
-\end{array}$ & $\begin{array}{c}.49 \\
.06 \\
21 / 36\end{array}$ & $\begin{array}{l}- \\
- \\
-\end{array}$ & $\begin{array}{l}- \\
- \\
-\end{array}$ \\
\hline
\end{tabular}

Note-For the 200- and 17-msec SOAs, for the 1st TOJ group, the data are the proportion of times the first-presented word was correctly selected, whereas for the 2 nd TOJ group, they are the proportion of times the second-presented word was correctly selected. For the $0-\mathrm{msec} \mathrm{SOA}$, the numbers are the proportion of times the primed item was selected as the correct response, even though there was no "correct" response. These 0-msec SOA columns are not aligned because the primed item and unprimed items were presented simultaneously, and do not fit with any of the other columns. For each SOA $(200$ and $17 \mathrm{msec}) \times$ Primed- vs. Unprimed-Word First cell, each participant contributed 40 observations. Each participant contributed 40 observations in the 0 -msec SOA cell. aFollowing Dunlap, Cortina, Vaslow, and Burke (1996), we computed Cohen's $d$ based on (1) the standard deviations of the scores within the primed and unprimed conditions, which assumes that priming is between subjects, $d_{\mathrm{IGD}}$ [which is $d_{4}^{\prime}$ in Cohen's (1988, p. 49) Eq. 2.3.8] and (2) the standard deviation of the individual within-subjects unprimed minus primed difference scores, $d_{\mathrm{CD}}$. Based on our (correlated) within-subjects $t$ (called $t_{\mathrm{C}}$ ), $d_{\mathrm{IGD}}=\sqrt{2}(1-r) \times t_{\mathrm{C}} / \sqrt{ } N$, where $r$ is the correlation for the within-subjects paired primed and unprimed RTs, and $d_{\mathrm{CD}}=\sqrt{2} \times t_{\mathrm{C}} / \sqrt{ } N$. Dunlap et al. recommend that $d_{\mathrm{IGD}}$ be used as a measure of effect size in metaanalyses. However, with respect to determining statistical power from Cohen's (chap. 2) tables, $d_{\mathrm{CD}}$ (what Cohen, p. 49, computes as $d$ in Eq. 2.3.9) should be used. For $N<40$, the power associated with $d_{\mathrm{CD}}$ in the tables will be slightly overestimated; for $N \geq 40$, the tabled power values will be very close to the true value (see Cohen, p. 49). bThese ratios represent the number of observers demonstrating a priming effect (in the same direction as the mean) out of the number of observers demonstrating a nonzero priming effect. This value is significantly different from chance $(.5), p<.05$, two-tailed. ${ }^{*} p<.05$, two-tailed.

priming also produces a response bias to guess the primed item as the correct response. Notwithstanding the clarity and novelty of our results, a number of issues need to be addressed in future research.

Empirically, one might ask why we found a contribution of response bias to the priming effect on TOJs, whereas Scharlau (2004) did not. The discrepant results could have been due to any of several procedural differences (ours vs. hers, respectively): (1) word versus simple shape stimuli, (2) the absence versus presence of an exogenous cuing manipulation, and (3) the absence versus presence of repeated TOJ stimuli across trials.

At the theoretical level, it remains to be determined exactly how priming influences the perception of temporal priority. Stolz (1999) suggested that the primed word was given attentional priority/precedence, which caused it to be perceived as occurring earlier. There are two distinct mechanisms that could mediate such an effect. First, priming could be causing a shift in spatial attention toward the primed word's location. However, this seems unlikely, given Stolz and Stevanovski's (2004) findings that exogenous cuing (which is known to cause shifts in spatial attention) affects the uptake of perceptual information, whereas priming seems not to (but see note 2). This suggests that exogenous cuing and priming affected different processing stages with their procedures. If Stolz and
Stevanovski's results (obtained when exogenous cuing was manipulated and a different task, lexical decision, was used) generalize to our procedures, then the priminginduced attentional precedence effect for our TOJs was likely not due to shifts in spatial attention. Of course, it is possible that when exogenous cuing is not being explicitly manipulated, priming does cause a shift in spatial attention. This is plausible because the control of spatial attention via exogenous cuing might preempt the control of spatial attention via priming. That is, after exogenous cuing has already drawn attention to a word's location, whether that word is related or unrelated to the prime may have no further effect on capturing spatial attention, though it can affect the disengagement of spatial attention (see Stolz, 1996).

A second account of how priming could influence perceived temporal priority during TOJs via attentional precedence would in no way appeal to shifts of spatial attention. Rather, the activation that priming produces could affect perception of the primed item's temporal priority either by reducing the latency for that item to enter consciousness or by reducing the amount of time it takes to process completely the identity/meaning of the primed word. By the latter processing completion account, observers would infer that the item for which processing first ends is the item that appeared first. Future research should attempt to 
distinguish between these two explanations of how priming affects the perception of temporal priority.

Notwithstanding the need for additional research to address these issues, the present results significantly advance our understanding of the processes underlying TOJs. Although it has previously been demonstrated both across the visual versus touch sensory modalities (e.g., Spence et al., 2001) and within the visual modality (e.g., Scharlau, 2004) that exogenous cuing influences TOJs via a perceptual prior-entry effect but not via a response bias, no such demonstration has previously been provided for priming, though priming has been shown to affect TOJs (Stolz, 1999). By providing a clear demonstration that the influence of priming on TOJs can be attributed to a true perceptual prior-entry effect, the present research sets the stage for future studies that compare and contrast the mechanisms that modulate prior-entry effects caused by priming and by the exogenous cuing of spatial attention. Such research would substantially promote our understanding of both the TOJ task, which has been extensively used in several research domains (see Spence et al., 2001), and attentional precedence, which is a fundamental component of cognition.

\section{REFERENCES}

CoHEn, J. (1988). Statistical power analysis for the behavioral sciences. Hillsdale, NJ: Erlbaum.

Dannenbring, G. L., \& Briand, K. (1982). Semantic priming and the word repetition effect in a lexical decision task. Canadian Journal of Psychology, 36, 435-444.

Dunlap, W. P., Cortina, J. M., Vaslow, J. B., \& Burke, M. J. (1996). Meta-analysis of experiments with matched groups or repeated measures designs. Psychological Methods, 1, 170-177.

JACOBY, L. L. (1991). A process dissociation framework: Separating automatic from intentional uses of memory. Journal of Memory \& Language, 30, 513-541.

Neely, J. H. (1977). Semantic priming and retrieval from lexical memory: Roles of inhibitionless spreading activation and limited-capacity attention. Journal of Experimental Psychology: General, 106, 226-254.

Posner, M. I. (1980). Orienting of attention. Quarterly Journal of Experimental Psychology, 32, 3-25.

SCHARLAU, I. (2002). Leading, but not trailing, primes influence temporal order perception: Further evidence for an attentional account of perceptual latency priming. Perception \& Psychophysics, 64, 13461360.

Scharlau, I. (2004). Evidence against response bias in temporal order tasks with attention manipulation by masked primes. Psychological Research, 68, 224-236.

Scharlau, I., \& Neumann, O. (2003). Perceptual latency priming by masked and unmasked stimuli: Evidence for an attentional interpretation. Psychological Research, 67, 184-196.

SCHNEIDER, W. (1995). Micro Experimental Laboratory (Version 2.0) [Computer software]. Pittsburgh, PA: Psychology Software Tools.
Spence, C., Shore, D. I., \& Klein, R. M. (2001). Multisensory prior entry. Journal of Experimental Psychology: General, 130, 799-832.

Stelmach, L. B., \& Herdman, C. M. (1991). Directed attention and perception of temporal order. Journal of Experimental Psychology: Human Perception \& Performance, 17, 539-550.

SternberG, S. (1969). The discovery of processing stages: Extensions of Donders' method. Acta Psychologica, 30, 276-315.

Stolz, J. A. (1996). Exogenous orienting does not reflect an encapsulated set of processes. Journal of Experimental Psychology: Human Perception \& Performance, 22, 187-201.

Stolz, J. A. (1999). Word recognition and temporal order judgments: Semantics turns back the clock. Canadian Journal of Experimental Psychology, 53, 316-322.

Stolz, J. A., \& McCanN, R. S. (2000). Visual word recognition: Reattending to the role of spatial attention. Journal of Experimental Psychology: Human Perception \& Performance, 26, 1320-1331.

Stolz, J. A., \& Neely, J. H. (1995). When target degradation does and does not enhance semantic context effects. Journal of Experimental Psychology: Learning, Memory, \& Cognition, 21, 596-611.

Stolz, J. A., \& STEVANOVSKI, B. (2004). Interactive activation in visual word recognition: Constraints imposed by the joint effects of spatial attention and semantics. Journal of Experimental Psychology: Human Perception \& Performance, 30, 1064-1076.

Titchener, E. B. (1908). Lectures on the elementary psychology of feeling and attention. New York: Macmillan.

\section{NOTES}

1. As is always so for the method of opposition, these predictions follow only under the assumption that the two processes (in this case, perceptual enhancement and response bias) operate to the same degree for both instructional sets. We know of no good reason to doubt the validity of this assumption in the present paradigm.

2 . When the validity of an exogenous cue in predicting the target's location is $80 \%$, priming and exogenous cuing do interact (Stolz \& McCann, 2000; Stolz \& Stevanovski, 2004). However, when cue validity is high, cuing has an endogenous component. Stolz and Stevanovski (pp. 1074-1075) provide a detailed conceptual analysis of why priming and endogenous cuing might interact. A second point is that when semantic priming is likely strategically mediated and the target always appears at fixation, the obtained interaction between the effects of priming and perceptual degradation of the target suggests that priming enhances early perceptual processing (see, e.g., Stolz \& Neely, 1995). But, once again, strategic priming is endogenous. Because the prime was not informative about which word would appear first in either Stolz's (1999) experiments or the present experiment, their priming effects are likely due to spreading activation and not to an endogenous strategic mechanism (cf. Neely, 1977).

3 . For the 0 -msec SOA, for the TOJ-1st group, the mean corresponds to the primed word 1 st means for the other SOAs because it refers to the probability of choosing the primed item (as having occurred first); for the TOJ-2nd group, the mean corresponds to unprimed word 1st means for the other SOAs because it refers to the probability of choosing the primed item (as having occurred second).

(Manuscript received March 16, 2005; revision accepted for publication November 23, 2005.) 